\title{
PREVALENCIA DE PATOLOGÍAS PULPARES EN PACIENTES ATENDIDOS EN LAS CLÍNICAS DE ENDODONCIA DE LA UNIVERSIDAD SAN GREGORIO DE PORTOVIEJO
}

\section{PREVALENCE OF PULP PATHOLOGIES IN PATIENTS TREATED AT THE ENDODONTIC CLINICS OF THE SAN GREGORIO DE PORTOVIEJO UNIVERSITY}

Thainah Bruna Santos Zambranoํㅗㄴ Lady Laura Parrales Espinoza², María Gabriela García Iturralde

1 Cirujana Dentista, Docente investigadora carrera de Odontología, Universidad San Gregorio de Portoviejo, Ecuador. https://orcid.org/0000-0002-8585-4763

Correspondencia:

2 Egresada carrera de Odontología. Universidad San Gregorio de Portoviejo, Ecuador. https://orcid.org/0000-0003-1422$\underline{2109}$

${ }^{3}$ Especialista en Endodoncia, Docente carrera de Odontología. thainahbruna@gmail.com Universidad San Gregorio de Portoviejo, Ecuador. https://orcid.org/0000-0002-8129-0972

Recibido: 11-11-2021

Aceptado: 23-12-2021

Publicado: 05-01-2022

\section{RESUMEN}

Objetivo: Determinar la prevalencia de patologías pulpares en pacientes atendidos entre 25 a 35 años en las clínicas de Endodoncia en la Universidad San Gregorio de Portoviejo (USGP) en el período 2015- 2017 mediante los registros de historia clínica archivados en la base de datos digital. Metodología: Estudio no experimental, transversal, descriptivo de corte retrospectivo, bibliográfico, de observación indirecta. Resultados: Las personas con mayor afectación pulpar registradas son el grupo etario de 28 y 30 años para pulpitis irreversible sintomática en el grupo masculino, contrario al grupo femenino para pulpitis irreversible asintomática a la edad de 28 años, en necrosis pulpar existió alto índice a los 34 años, así como daños por patologías pulpares en la pieza \#21, \#12, \#22. Conclusiones: La alta prevalencia de patologías pulpares depende de la falta de conocimiento y de compromiso por parte de las personas al no acudir de manera oportuna al profesional de salud al presentar sintomatología por lo que ocurren complicaciones e incluso pérdida dental generando problemas en el sistema estomatognático.

Palabras claves: endodoncia, necrosis pulpar, pulpitis.

\section{ABSTRACT}

Objective: to determine the prevalence of pulpal pathologies in patients attended between 25 and 35 years of age in the Endod ontics clinics at the Universidad San Gregorio de Portoviejo (USGP) in the period 2015- 2017 by means of the clinical history records filed in the digital database. Methodology: non-experimental, cross-sectional, descriptive, retrospective, bibliographic, indirect observation study. Results: the people with the greatest pulp involvement recorded are the age group of 28 and 34 years for symptomatic irreversible pulpitis in the male group, contrary to the female group within the same age range for pulp necrosis, this in pieces 11 and 12, where there is a higher prevalence for the first pathology. Conclusions: The problems depend on the lack of knowledge and commitment on the part of the people by not going to the health professional in a timely manner when presenting symptoms, which is why most of them have complications and even dental loss, generating problems in the stomatognathic system.

Keywords: endodontics, pulp necrosis, pulpitis, pulpitis

\section{INTRODUCCIÓN}

La pulpa dental es un tejido conectivo semejante a cualquier otro del organismo humano, integrada por células, sustancia fundamental amorfa, componentes vasculares, linfáticos y nerviosos, localizado en el interior de diente, proporcionado vitalidad al mismo, sus funciones principales son de formación y defensa, no obstante, el complejo pulpo dentinario es afectado de manera multifactorial por diversas causas que tienen como resultado inflamación, muerte pulpar, distrofia pulpar, entre las más comunes esta la caries, traumatismo e iatrogenia. ${ }^{1,2}$

El conocimiento de la morfología dental es imprescindible en el tratamiento endodóntico especialmente en las características anatómicas internas del diente, de sus cámaras donde se inserta la pulpa coronaria y conductos radiculares donde se aloja la pulpa radicular, en los dientes anteriores la 


\section{REVISTA CIENTÍFICA “ESPECIALIDADES ODONTOLÓGICAS UG”. ISSN: 2600576X ÓRGANO OFICIAL DE LA FACULTAD PILOTO DE ODONTOLOGÍA DE LA UNIVERSIDAD DE GUAYAQUIL}

parte coronaria tiene cuatro caras y un borde incisal (o techo), la mayoría poseen un solo conducto, mientras que los dientes posteriores existen cuernos pulpares, piso pulpar y poseen dos a tres conductos. ${ }^{3}$

Las enfermedades pulpares son un verdadero reto para la odontología, es la respuesta de la pulpa ante un irritante a la que de manera inicial se adapta y luego se resiste, resolviendo la leve lesión o contrario a ello una reacción violenta. ${ }^{4}$ La infección puede llegar a la pulpa mediante la corona o la raíz del diente, las caries, traumatismo y defectos del desarrollo dentario son las causas frecuentes de infección por el área de la corona, mientras que por la raíz son las caries cervicales, bolsas periodontales y bacteremias. ${ }^{5}$

La asociación Americana de Endodoncia realizo una guía de diagnóstico clínico para patologías pulpares y periapicales, que tienen como utilidad el recomendar de forma sistemática a los profesional y pacientes a tomar decisiones en cuanto a la atención sanitaria más apropiada en consecuencia el plan de tratamiento idóneo, interviniendo en su condición clínica específica e individualizada, con el objetivo de reconocer la parte patológica es necesario abordar lo fisiológico, la pulpa clínicamente normal se evidencia como el tejido pulpar libre de sintomatología que responde de manera normal a las pruebas de sensibilidad, en cuanto a la pulpitis reversible es caracterizado de leve a moderada intensidad, donde no existe antecedentes antes los estímulos térmicos, la duración al dolor es de segundos. ${ }^{6}$

La pulpitis irreversible es un proceso agudo (intenso dolor) o crónico (presencia de pus) por un proceso de inflamación no tratada, en ambos estados la pulpa se encuentra vital y en ella se encuentra el proceso inflamatorio de manera activa aun retirando el estímulo, de acuerdo a la sintomatología, la pulpitis irreversible sintomática se caracteriza por dolor prolongado especialmente al calor, contrario a la pulpitis irreversible asintomática no presenta síntomas relevantes y puede avanzar hacía necrosis pulpar es decir la muerte de la pulpa dental. ${ }^{7}$
La necrosis pulpar es la muerte del tejido pulpar, se clasifica en total o parcial esto en dependencia de la porción afectada de la pulpa, su etiología tiene como causa la inflamación y el traumatismo, situación en la que la pulpa es destruida antes de que ocurra una reacción inflamatoria, traduciéndose en un infarto isquémico y causar una pulpa gangrenosa seca. ${ }^{8}$

El tratamiento endodóntico se resume en dos tipos de pulpectomía que pueden realizarse como biopulpectomía, la cuál es la técnica empleada en dientes con vitalidad pulpar, en patologías como pulpitis aguda, fracaso de tratamiento de pulpotomía o sangrado excesivo de apariencia rojo oscuro, reabsorción interna y exposición pulpar durante 1-2 días después de ocurrido un traumatismo. ${ }^{9}$ En el caso de la necropulpectomía se aplica cuando la pulpa dental se ve afectada de manera irreversible, donde ha ocurrido una necrosis pulpar sin lesión periapical, esta técnica se resume en al menos tres pasos: colocación de una a dos gotas de EDTA, instrumentación alrededor del conducto, irrigación con hipoclorito de sodio al $1 \%{ }^{10}$

\section{MATERIALES Y MÉTODOS}

Se elaboró un estudio cuantitativo, aplicando criterios no estadísticos, las unidades de análisis estuvieron conformadas por 51 historias clínicas de pacientes atendidos en las clínicas de Endodoncia de la Universidad San Gregorio de Portoviejo entre 25-35 años de edad en el período 2015-2017, con la finalidad de describir el objeto de estudio. El diseño de la investigación es no experimental, transversal, descriptivo, de corte retrospectivo, bibliográfico, de observación indirecta.

\section{RESULTADOS}

En estos resultados se encontró que el grupo mayormente afectado fue el estrato masculino representado por el $35 \%$ en rango de edad de 28 y 30 años ambos con un 50\%, en los demás individuos no se registró cifras para pulpitis irreversible sintomática. (Gráfico 1)

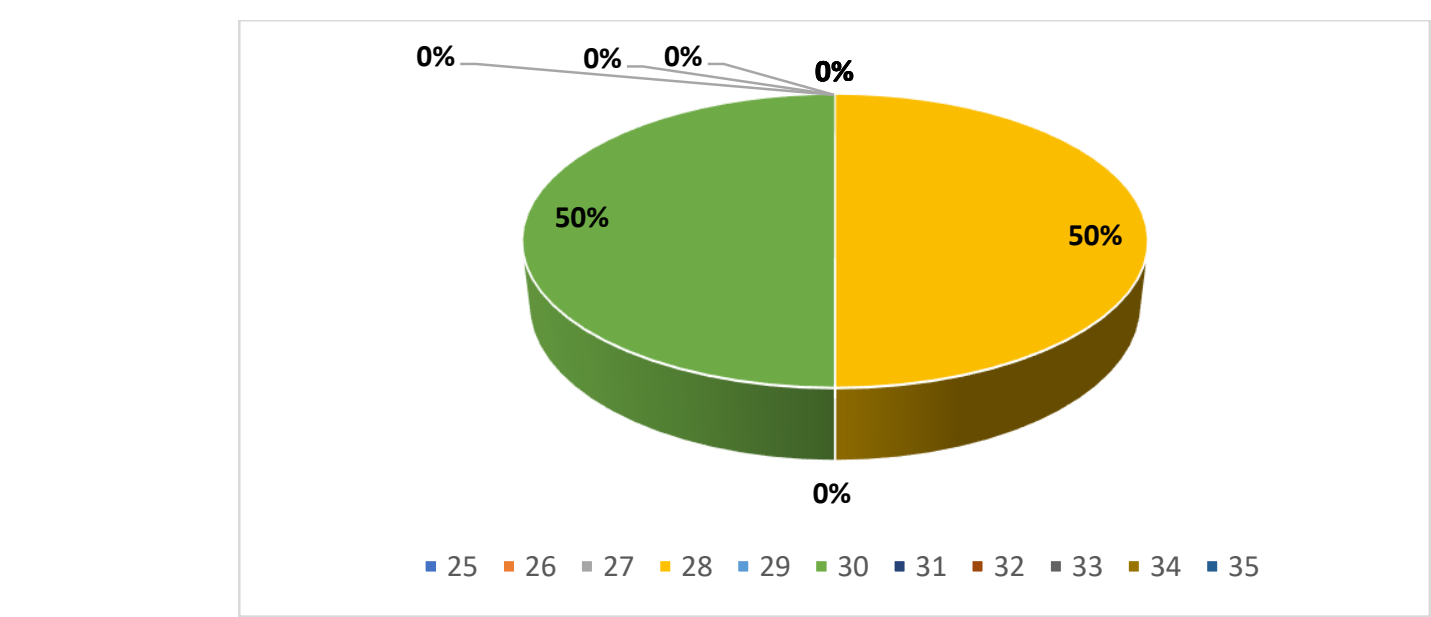

Gráfico 1. Distribución de la muestra de acuerdo al grupo etario del grupo masculino en personas entre 25-35 años en pulpitis irreversible sintomática. 


\section{REVISTA CIENTÍFICA “ESPECIALIDADES ODONTOLÓGICAS UG". ISSN: 2600576X ÓRGANO OFICIAL DE LA FACULTAD PILOTO DE ODONTOLOGÍA DE LA UNIVERSIDAD DE GUAYAQUIL}

En el grupo femenino conformado por $65 \%$ la patología pulpar prevaleciente es la pulpitis irreversible asintomática en la edad de 28 años con un 44\%, seguido de los 34 años con un $22 \%$ y por último, 33 y 35 años con un $11 \%$. (Gráfico 2).

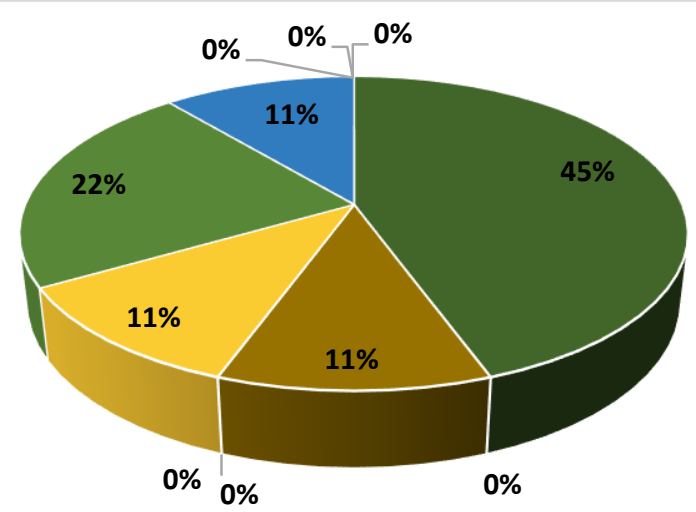

$=25 \quad 26 \quad-27 \quad-28 \quad-29 \quad-30 \quad-31 \quad-32 \quad-33 \quad-34 \quad-35$

Gráfico 2. Distribución de la muestra en el grupo femenino de personas entre 25-35 años para pulpitis irreversible asintomática.

De acuerdo al estudio el grupo femenino fue más afectado por necrosis pulpar que el masculino en edad de 34 años con un $31 \%$ luego de esto en edades de 26, 29 y 32 años de edad con un 13\%, en minoría a los 25, 30, 31, 33, 35 con un 6\%. (Gráfico 3)

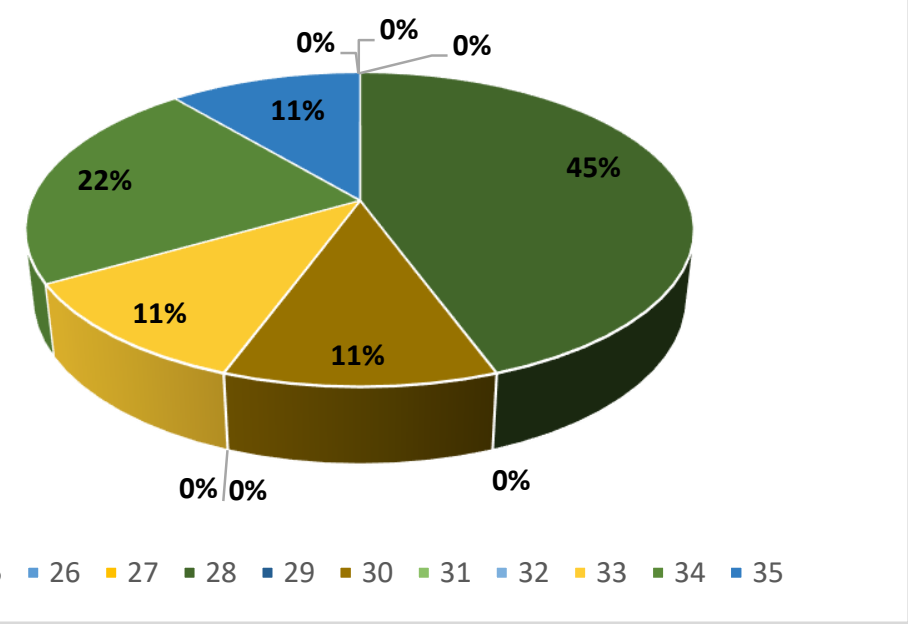

Gráfico 3. Distribución de la muestra en el grupo femenino de personas entre 25-35 años para necrosis pulpar

Para necrosis pulpar en el estudio la pieza número \#21 fue mayormente afectada a los 34 años con un 67\%, seguido del grupo de personas compuesto por 28 años con un 33\%. (Gráfico 4)

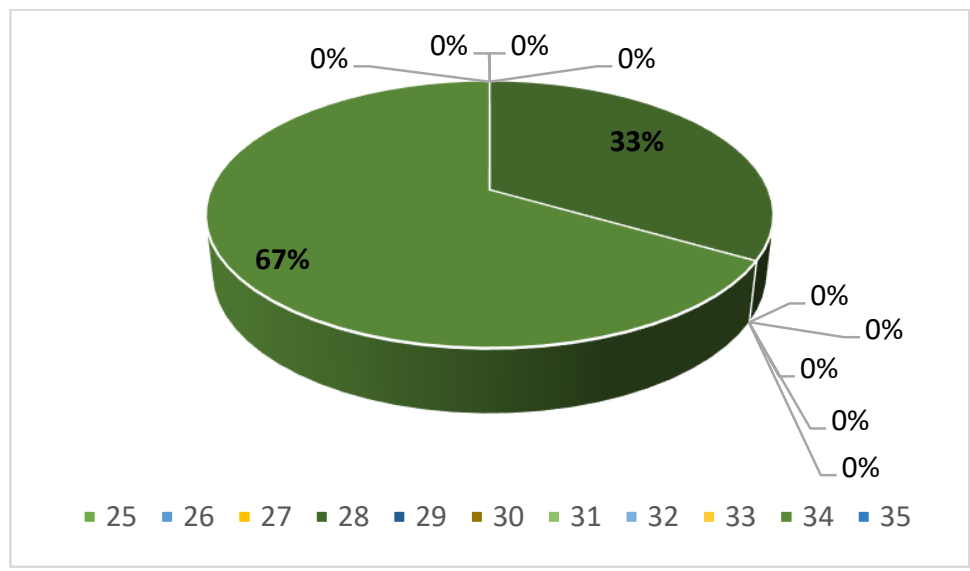

Gráfico 4. Pieza dental mayormente afectada para necrosis pulpar en individuos entre 25-35 años de edad. 


\section{REVISTA CIENTÍFICA “ESPECIALIDADES ODONTOLÓGICAS UG”. ISSN: 2600576X \\ ÓRGANO OFICIAL DE LA FACULTAD PILOTO DE ODONTOLOGÍA DE LA UNIVERSIDAD DE GUAYAQUIL}

La pieza dental número \#12 fue la segunda mayormente afectada en el grupo de personas entre 25-35 años para necrosis pulpar (Gráfico 5)

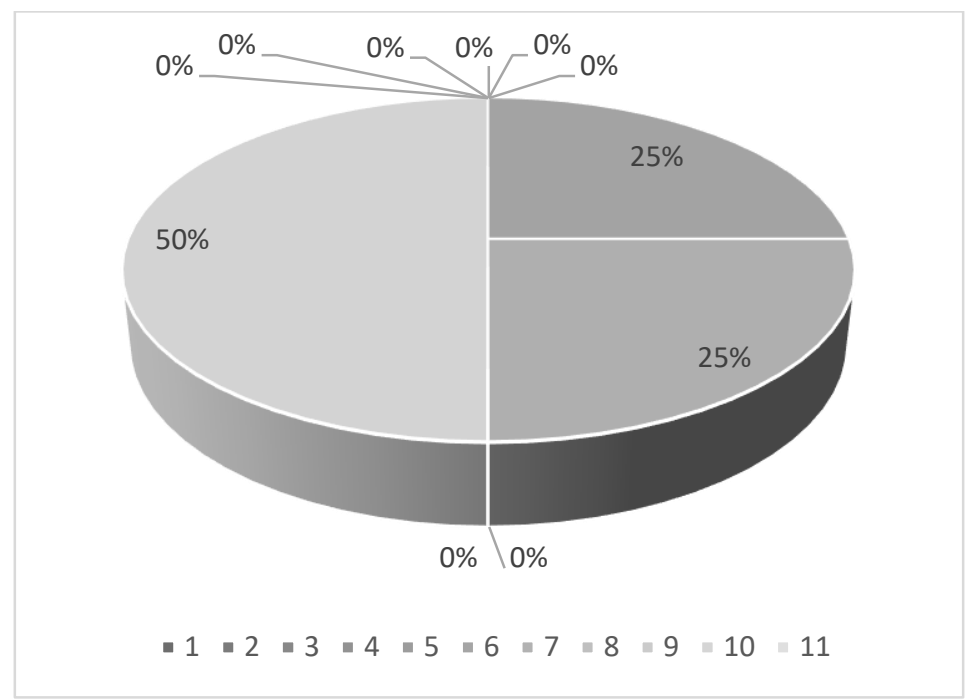

Gráfico 5. Segunda pieza dental mayormente afectada para necrosis pulpar en individuos entre 25-35 años de edad

Finalmente, la pieza dental \#22 fue mayormente afectada en el estudio por pulpitis irreversible ya sea reversible o irreversible, en edades de 34 y 35 años con un $25 \%$ y con un $13 \%$ edades como 28 y 30 años. (Gráfico 6 )

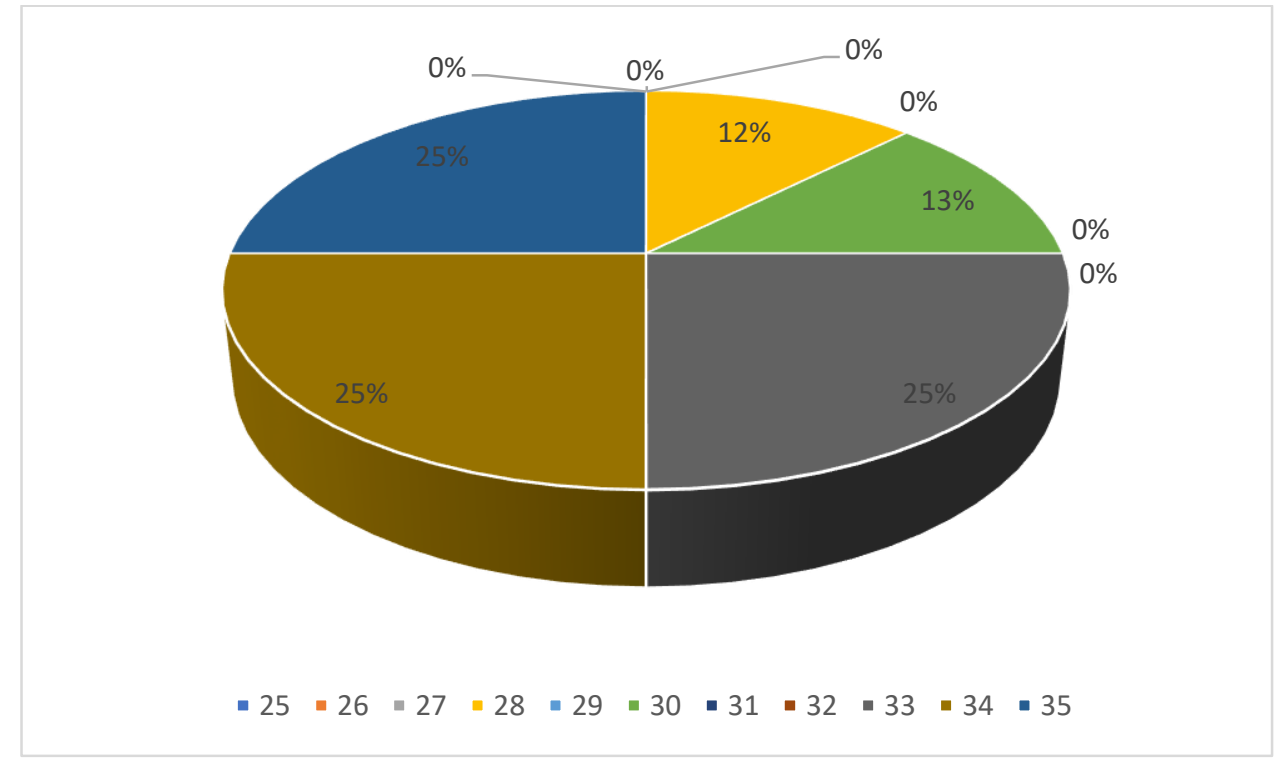

Gráfico 6. Pieza dental mayormente afectada para pulpitis en individuos entre 25-35 años de edad.

\section{DISCUSIÓN}

En el grupo estudiado se presentaron los siguientes problemas en relación a la presencia de patologías pulpares:

En cuanto al grupo femenino las patologías pulpares que mayormente afectaron son la pulpitis irreversible asintomática en el rango de 28 años y necrosis pulpar en el grupo de 34 años, así mismo existió mayor prevalencia de pulpitis irreversible sintomática en el estrato masculino en edades entre 28 y 30 años. De manera general las piezas mayormente afectadas son la \#12, \#21 y \#22, estos resultados están en similitud con los de Alberto Balcázar donde menciona que en el género masculino la enfermedad pulpar es baja y es predisponente las periapicales, ${ }^{11}$ no obstante, existe diferencias al señalar en el mismo artículo que la pieza mayormente afectada fue la \#36 y \#46, hecho que es explicado al ser el segmento anterior las piezas dentales que reciben tratamiento endodóntico en la USGP.

En un estudio realizado en México, la enfermedad pulpar más frecuente fue la pulpitis irreversible en ambos géneros, hechos que mantienen relación con nuestro estudio al ser el sexo masculino y femenino afectados por pulpitis irreversible sintomática y asintomática12, de igual manera en un estudio 


\section{REVISTA CIENTÍFICA “ESPECIALIDADES ODONTOLÓGICAS UG". ISSN: 2600576X ÓRGANO OFICIAL DE LA FACULTAD PILOTO DE ODONTOLOGÍA DE LA UNIVERSIDAD DE GUAYAQUIL}

realizado en Brasil como patología predominante se observó la pulpitis sintomática, misma patología observada en el grupo masculino de 28-30 años de edad. ${ }^{13}$

En el estudio realizado por Najarro ${ }^{14}$ el sexo femenino fue mayormente afectado lo cual no concuerda con lo obtenido, esto porque en la presente investigación el grupo mayormente afectado fue el masculino, situación que se justifica por el número de personas implicadas en ambos estudios.

Los resultados concuerdan con el de Ferrer donde predomino las enfermedades pulpares con un 53,80\% mientras que el estudio esta prevaleció con un $50 \%$, no obstante, el grupo afectado mayormente fue el de 35-59 años contrario al señalado por los autores donde se menciona que fue en el grupo etario de 28-30 años, estos grupos son mayormente afectados pues se encuentran en etapa estudiantil y laboral y por tanto la atención odontológica queda en abandono. ${ }^{15}$

Se recomienda darle continuidad al estudio con un mayor número de participantes, esta investigación contribuye a los profesionales al mostrar altos índices de patologías pulpares y el impacto que este tiene en la vida de cada individuo, que significa un problema de salud pública el mismo, debe priorizarse como prevención a través de campañas preventivas de salud bucodental a la comunidad y de esta forma disminuir el índice de enfermedades pulpares, pérdida de dientes o incluso lesiones endoperiodontales, recordando que el foramen apical es la vía principal de comunicación entre la pulpa y el periodonto que permite el ingreso de elementos inflamatorios causando una patología periapical, de esta manera compromete al periodonto. 16

\section{CONCLUSIONES}

Se determina que es importante realizar una evaluación clínica y radiográfica cuya finalidad es obtener resultados favorables en diagnóstico y plan de tratamiento. Existe un alto índice de enfermedades pulpares por el desconocimiento y la poca importancia que algunas personas prestan a la salud oral. Recordemos que al pasar el tiempo la situación del paciente se complica aún más y el número de problemas será mayor, esto en patologías periapicales, lesiones endoperiodontales, pérdida de piezas dentales y posteriores, impacto en la oclusión y en el sistema estomatognático de manera general.

\section{REFERENCIAS BIBLIOGRÁFICAS}

1. Stock C, Gulabívala K, Walker R, Goodman J. Atlas en color y texto de endodoncia. Segunda edición. Madrid: Harcourt Brace;1996.

2. Hargreaves K, Cohen S, Berman L. Vías de la pulpa. Décima edición: España: Elsevier Mosby;2011.

3. Rodríguez A. Endodoncia. Consideraciones actuales. Primera edición. Colombia: Amolca; 2003

4. Montoro Y, Fernández M, Vila D, Rodríguez A, Mesa D. Urgencias estomatológicas por lesiones pulpares. Rev
Cubana Estomatol [Internet]. 2012. [consultado 28 septiembre 2021]; 49(4): 286-294. Disponible en: http://scielo.sld.cu/scielo.php?script=sci arttext\&pid= S0034-75072012000400004\&lng=es.

5. López J. Etiología, clasificación y patogenia de la patología pulpar y periapical. Med oral Patol Oral Cir Bucal. 2004[Consultado 28 septiembre 2021]; 9. Suppl: S52-62. Disponible en: http://www.medicinaoral.com/medoralfree01/v9Supp li/medoralv9supplip58.pdf

6. Marroquín T, García C. Guía de diagnóstico clínico para patologías pulpares y periapicales. Versión adaptada y actualizada del "consensus conference recommended diagnostic terminology", publicado por la asociación americana de Endodoncia (2009). Rev. facultad de odontología Universidad de Antioquía. 2015. [Consultado 28 septiembre 2021] 26 (2):1-27.

7. Murillo H, Bustamante C. Pulpitis irreversible. Rev. boliviana [Internet]. 2012 [Consultado 28 septiembre 2021] 21 (7): 1-6. Disponible en: http://www.revistasbolivianas.org.bo/pdf/raci/v21/v2 1a07.pdf

8. Vilchis S, Gurria A, Rodríguez A, Treviño R. Necrosis pulpar con lesión periapical. Rev. Mexicana de Estomatología .2018. [Consultado 28 septiembre 2021]. 5 (2): 1-6. Disponible en: https://www.remexesto.com/index.php/remexesto/art icle/view/231/413

9. Mendoza A, Valencia S. Pulpectomia. Rev. Act. Clin. Med [Internet]. 2012. [consultado 28 septiembre 2021]. 23:15 Disponible en: http://www.revistasbolivianas.org.bo/scielo.php?script =sci arttext\&pid $=$ S230437682012000800007\&lng=es.

10. Ramos P, Rosales G. Limpieza y obturación del sistema de conductos: biopulpectomía, necropulpectomia y técnica de condensación lateral modificada. La Candonia. 2017. [Consultado 28 septiembre 2021]. Disponible en: https://d1wqtxts1xzle7.cloudfront.net/38528849/291 021PBwithcoverpagev2.pdf?Expires $=1632885551 \&$ Sig nature $=$ Ld8Xh0JSn2b5ywtCwlAlRYx3H69u2zQT0XfRW ح YaTWBNw4WAfN27mek41CwwaB8Dn62uSvV2LOTQ FsRUy m09Dsyi69lqKizholVbeL 9x0B0syKoPZAKQFz 2Nmyh7FzCg10ECO2bKEWdJs0SAVObEbG0CWh69sNU phjZCP8c0R9GS3Ud3WIf7bnxyKuz79CWI5aSOmzDMz8 QV2sG4mTXyiUzhJqok0Egkiuk 53t02tHb JK60LhoGO vu4t0rSzY5bU7IRtxtAShHZd5btPnKQcq3tZyg31pmfs6x Nh UP8Mt0Et1jBIcXRDANo1vVID80I6KIgiqyS0jyiA \& Key-Pair-Id=APKAJLOHF5GGSLRBV4ZA

11. Balcázar C, Isidro L, Nájera A, Hernández G, Rueda M, Garrido S. Factores de riesgo asociados a la patología periapical y pulpar. Horiz. sanitario [Internet]. 2017 [consultado 01 octubre 2021]; 16(2): 111-119. Disponible en:

http://www.scielo.org.mx/scielo.php?script=sci_arttext $\underline{\text { \&pid=S200774592017000200111\&lng }}$ 


\section{REVISTA CIENTÍFICA “ESPECIALIDADES ODONTOLÓGICAS UG”. ISSN: 2600576X ÓRGANO OFICIAL DE LA FACULTAD PILOTO DE ODONTOLOGÍA DE LA UNIVERSIDAD DE GUAYAQUIL}

12. Soberaniz V, Alonso L, Vega E. Frecuencia de patología pulpar en la clínica hospital de petróleos Mexicanos Coatzacoalcos, Veracruz. Rev. científica odontológica [Internet] 2012 [Consultado 1 octubre 2021] Disponible en:

https://revistaodontologica.colegiodentistas.org/index. $\mathrm{php} / \mathrm{revista/article/view/446/661}$

13. Estrela C, Aguirre O, Almeida J, Rodríguez C, et al. Diagnostic and clinical factors associated with pulpal and periapical pain. Rev. Braz. Dent. J. [Internet]2011. [Consultado el 1 octubre 2021] 22 (4):306-3011. Disponible en:

https://www.scielo.br/i/bdj/a/Rhw5Wk465Dr9SY4xL ZwMfyf/?format=pdf\&lang=en

14. Najarro E, Montero C, Mentado L, Ferrer D. Enfermedades pulpares y periapicales en el servicio de urgencias. Rev. Inmedsur. [Internet] 2021. [Citado 1 de octubre 2021] 4 (1): e143. Disponible en: http://www.inmedsur.cfg.sld.cu/index.php/inmedsur/ article/view/143/116

15. Ferrer D, Hernández A, García O, Rodríguez Y, Pérez M, Liriano R. Caracterización de las enfermedades pulpares en pacientes pertenecientes al área II del municipio Cienfuegos. Rev. Medigraphic [Internet] 2017 [Citado 1 octubre 2021]; 15 (3):1-6. Disponible en: https://www.medigraphic.com/pdfs/medisur/msu2017/msu173g.pdf

16. Pesqueira $P, \quad$ Carro H. Lesiones endoperiodontales. Odontología Vital. Rev. Scielo [Internet]. 2017 [consultado el 1 octubre 2021]; (27): 35-44. Disponible en: http://www.scielo.sa.cr/scielo.php?script=sci_arttext\& pid=S165907752017000200035\&lng=en.

\section{Conflictos de intereses}

Los autores señalan que no existe conflicto de intereses durante la realización del estudio, no se recibió fondos para la realización del mismo, el presente solo fue sometido a la Revista Científica "Especialidades odontológicas UG" para su revisión y publicación

\section{Financiamiento}

Los autores indican la utilización de fondos propios para la elaboración del trabajo de investigación.

\section{Declaración de contribución}

Todos los autores han contribuido en elaboración del trabajo de investigación, en las diferentes partes del mismo. 